\title{
Spontaneous cervical emphysema: a challenge in establishing a plausible etiology
}

\author{
Andrew C.G.J' ${ }^{1}$ Ahmed N.A ${ }^{2}$, Hamal M.H ${ }^{3}$ \\ ${ }^{1}$ Dr. Andrew CGJ, ${ }^{2}$ Dr. Ahmad NA, ${ }^{3}$ Dr. Hamal MH, all authors are affiliated with Otorhinolaryngology Department, \\ Hospital Queen Elizabeth, Kota Kinabalu, Sabah, Malaysia
}

Address for Correspondence: Dr. Andrew Charles Gomez Junior, Otorhinolaryngology Department, Hospital Queen Elizabeth, Kota Kinabalu, Sabah, Malaysia. Email: aj06556@hotmail.com

\begin{abstract}
Spontaneous cervical emphysema occurs in the absence of previous disorders or intiating factors and often poses a challenge in establishing an etiology at presentation. We report a 23 year old lady who presented with extensive subcutaneous emphysema in which a clear etiology was not evident during the initial presentation.
\end{abstract}

Keywords: Spontaneous Emphysema, Cervical Emphysema, Airway Obstruction.

\section{Introduction}

Cervical emphysema is clinical entity that may compromise the airway. It may occur as a complication of surgery within the anatomical regions of the neck, a breach to the integrity of the upper aero digestive tract, infection with a gas-forming organism or from direct externa trauma [1]. Spontaneous cervical emphysema occurs in the absence of previous disorders or initiating factors and often poses a challenge in establishing an etiology at presentation ${ }^{[2]}$. We report a 23 -year-old lady who presented with extensive subcutaneous emphysema in which a clear etiology was not evident during the initial presentation.

\section{Case Report}

A 23-year-old lady presented with right neck swelling associated with dysphagia and odynophagia and voice change for 4 days duration. Further history revealed a preceding right-sided toothache 1 week prior to presentation. Patient denied history of fever, drooling of saliva or dyspnea. She also had no symptoms suggestive of tuberculosis or its contact and denied asthma. General examination revealed a well looking, afebrile patient with a muffled voice. She was not in respiratory distress nor stridorous. Neck examination demonstrated a non-tender, fluctuant right neck swelling $6 x 6 \mathrm{~cm}$, with extensive subcutaneous emphysema extending from the angle of the mandible until the upper thorax as evidenced by crepitus. Oral exam showed marked trismus with generalized decayed teeth and a raised floor of mouth. Medialization of right lateral pharyngeal wall was seen with no evidence of peritonsilar bulge. Flexible nasopharyngolaryngoscopy confirmed the medialization of the right lateral pharyngeal and showed a swollen epiglottis, which did not appear erythematous, or inflamed. Bilateral vocal cords were mobile with no phonation gap. All other supraglottic stuctures were normal and the airway patent. With a white cell count of 31 thousand, a provisional diagnosis of right parapharyngeal abcess was deduced. Contrast enhanced computer tomography of the neck revealed extensive subcutaneous emphysema in bilateral parapharyngeal more on the right, right supraclavicular, and retropharyngeal regions [Fig 1]. No obvious collection was seen. After a dental review ruled out a dental origin for the current clinical condition, patient underwent a right neck exploration. Intraoperatively large gas pockets were found in the deep neck spaces containing small amounts of pus. These were opened and gas released. Cultures taken intraoperatively found no growth of organisms however a prior blood culture grew staphylococcus hominis. The patient was then transferred 
postoperatively to the intensive care unit for further monitoring and antibiotics. Patient was discharged later.

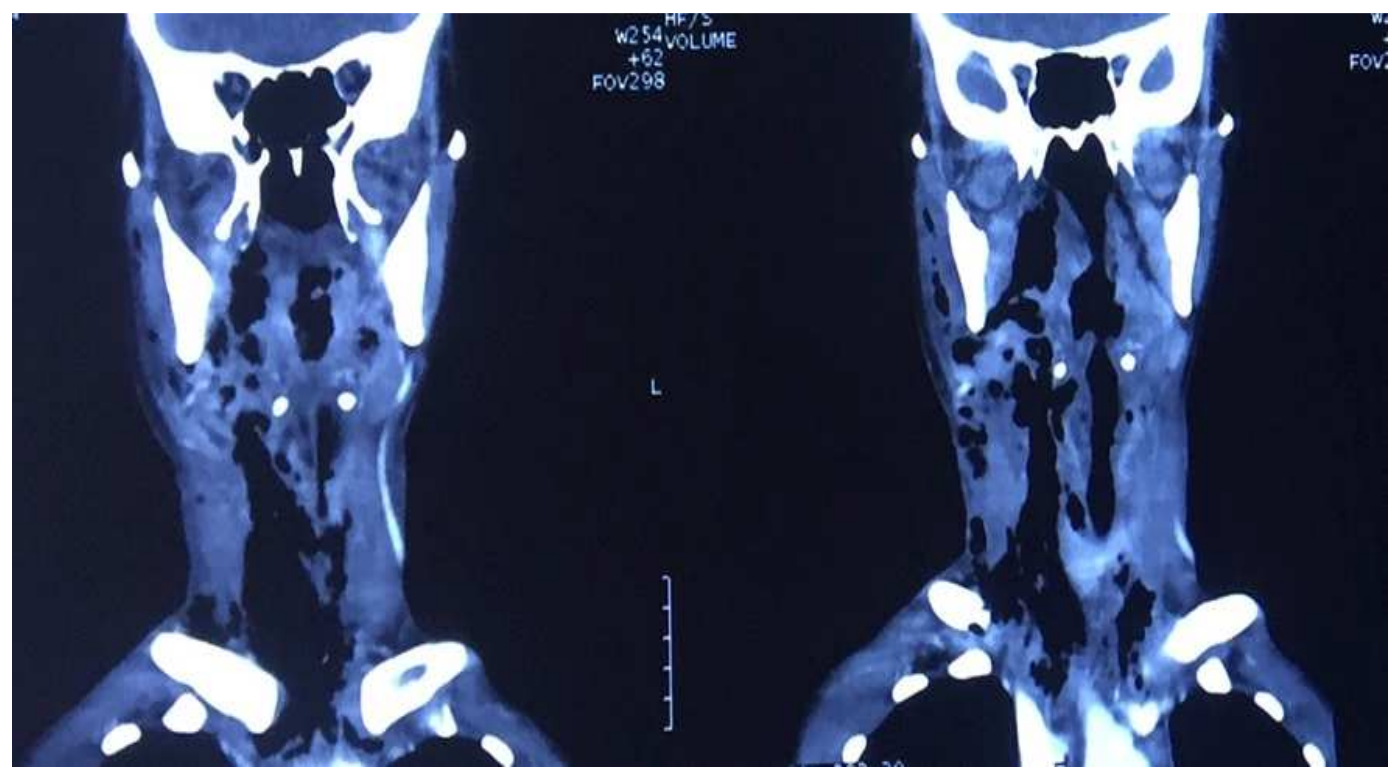

Figure-1: Coronal view of a contrast enhanced Computer tomography of the cervical region showing extensive subcutaneous emphysema in bilateral parapharyngeal more on the right and right supraclavicular regions

\section{Discussion}

Cervical emphysema is usually seen in association with penetrating trauma, infections from gas forming organisms, or cases in which a breach in the integrity of the upper aerodigestive tract has occurred [1,2]. In cases where these are not clinically evident, the term spontaneous cervical emphysema is used. Causes of spontaneous cervical emphysema include cases in which there is an increase in intrathoracic pressure [3].

These claim majority of the cases and are seen in patients needing positive pressure ventilation, deep-sea divers, labour, excessive explosive coughing and blowing [4,5]. Other causes include pulmonary conditions such as bronchiolitis, pneumonia and asthma or rupture of pulmonary bulla $[1,3,4]$. Surgically induced spontaneous emphysema has also been reported in cases of tooth extraction and gastrointestinal surgery in which the primary operated site is not within the cervical region [2, 5]. Dekel et al reported that the primary complaints of subcutaneous emphysema were neck swelling which may be associated with pain and also obstructive symptoms depending on the extent of the disease as in this case [5]. The diagnosis is evident clinically if crepitus is demonstrated over the cervical region in the absence of history or clinical findings of a plausable etiology. This is then confirmed with imaging either plain radiograph of the cervical region or as in our case with a computer tomography scan.
Management of such cases will depend greatly on the underlying disease process and degree of airway compromise [2,5]. This however usually involves surgical release of the emphysema and the advice against acts that may increase intrathoracic pressure including straining and coughing postoperatively so as to prevent recurrence. Rationale use of antibiotics should also be exercised as in this case.

\section{Conclusion}

Sponaneous cervical emphysema is a rare clinical entity in which a plausable etiology is not grossly apparent. A clinical finding of crepitus over the cervical region with no obvious cause from history and physical examination suggests its diagnosis. This is confirmed by imaging studies, which will also demonstrate its extent and may reveal an underlying disease process. Treatment usually involves surgical release with advice postoperatively to prevent its reoccurrence.

Note: This case was presented as a poster at the 18th Asian Research Symposium in Rhinology and in conjunction with 8th Malaysian International Congress on Otorhinolaryngology, 26th - 28th May 2016, Kuala Lumpur. Poster \#131

No author has any beneficial disclosures relevant to this article or conflict of interest to declare. 


\section{Acknowledgement}

Dr. Chong Hon Syn (MBBS, MS ORL-HNS [Mal])

Dr. Ong Cheng Ai (MBBS, MS ORL-HNS [Mal])

Dr. Halimuddin Bin Sawali (MBBS, MS ORL-HNS [Mal])

Dr.Yong Doh Jeing (MBBS, MS ORL-HNS [Mal], MRCS [Eng]).

Funding: Nil, Conflict of interest: None.

Permission of IRB: Yes

\section{Reference}

1. Maithani T, Dey D, Sharma C. Spontaneous cervicofacial subcutaneous emphysema secondary to pneumomediastinum in an otherwise asymptomatic child. Ear, Nose \& Throat Journal 2012;91(10):E9-E11.
2. Adekunle D, Adekunle OP. Retropharyngeal emphysema: An unusual aetiology - A case report and literature review. CRCM Case Reports in Clinical Medicine 2013;02(03):194-197.

3. Rasheed Z, Haris K. Subcutaneous emphysema in a case of infective sinusitis: A case report. Journal of Medical Case Reports 2010;4(1):235 .

4. Singla M, Potocko J, Sanstead J, Pepper P.Ooh-rah! An unusual cause of spontaneous pneumomediastinum. Military Medicine 2012; 177(11):1396-8.

5. Dekel B, Paret G, Szeinberg A, Vardi A, Barsilay Z. Spontaneous Pneumomediastinum in children; clinical and natural history 1996; 155:695-7.

\section{How to cite this article?}

Andrew C.G.J, Ahmed N.A, Hamal M.H. Spontaneous cervical emphysema: a challenge in establishing a plausible etiology. Int J Med Res Rev 2016;4(11):1943-1945.doi:10.17511/ijmrr. 2016.i11.06. 